\title{
Quality of life in individuals with tinnitus with and without hearing loss
}

Gregorina Silva Ribeiro Rocha(1)

Marlizete Maldonado Vargas ${ }^{(1)}$

Margarete Zanardo Gomes ${ }^{(1)}$

(1) Universidade Tiradentes, UNIT, Aracaju, Sergipe, Brasil.

Conflict of interest: Nonexistent

Received on: April 05, 2017

Accepted on: September 28, 2017

Mailing address:

Gregorina Silva Ribeiro Rocha

Instituto de Pesquisa e Tecnologia - ITP

Avenida Murilo Dantas, 300 - Farolândia

CEP: 49032-490 - Aracaju, Sergie, Brasil

E-mail: gregorinarocha@gmail.com

\section{ABSTRACT}

Purpose: to evaluate the perception of the level of quality of life in subjects with tinnitus, with and without hearing loss.

Methods: a cross-sectional quantitative study. A total of 189 subjects (mean age 53.06 years) divided into four groups. Group 1: subjects with normal auditory thresholds without tinnitus complaint; Group 2: subjects with normal auditory thresholds and tinnitus complaint; Group 3: subjects with sensorineural hearing loss without tinnitus complaint; Group 4: subjects with sensorineural hearing loss and with tinnitus complaint. Levels of quality of life were investigated through the World Health Organization Quality Of Life (WHOQOL) website - brief and psycho-emotional and functional aspects of patients with tinnitus through the Tinnitus Handicap Inventory (THI). Statistical analyzes, comparisons among groups and descriptive analysis were performed, considering a significance level of $5 \%$.

Results: the overall mean scores of quality of life in group 4 (56.07) were smaller than those of group $1(64.67)(p<0.05)$. The subjects with tinnitus complaint presented a moderate level of disturbance of the symptom.

Conclusion: tinnitus interferes in the quality of life of individuals who had preserved or altered auditory thresholds. Therefore, means to reduce the discomfort caused by tinnitus symptom should be developed, in order to improve patients' quality of life.

Keywords: Quality of Life, Tinnitus, Hearing, Hearing Disorders 


\section{INTRODUCTION}

Tinnitus is an auditory sensation perceived by the individual that is not originated from the external environment ${ }^{1}$. A study conducted in a South Indian population revealed that the most prevalent etiological factors leading to the development of tinnitus associated with hearing loss are otitis media $(60.9 \%)$, presbycusis (16.6\%) and otosclerosis (14.3\%). The authors also verified that in $96.9 \%$ of subjects with tinnitusthere was an association with hearing loss ?

About $15 \%$ of the world population (278 million) presents tinnitus ${ }^{3}$. In the United States, $10 \%$ of the population ( 30 million) has tinnitus ${ }^{4}$, and one in 10 adults presents the symptom ${ }^{5}$. In Brazil, it is estimated to find it in $13 \%$ of the population ( 28 million) ${ }^{3}$. In the city of São Paulo, São Paulo, Brazil, the prevalence is of $22 \%$, and the symptom affects mostly women ${ }^{6}$.

Concerning to the hearing loss, a study showed that it has been decreasing in North American adults aged between 20 and 69 years. It was found that the prevalence of hearing loss reduced from 16 to $14 \%$, i.e., from 28 million adults inthe years 1999-2004 to 27.7 million in the years 2011-20127.

Researchers evaluated 68 individuals with sensorineural hearing loss and tinnitus complaint from the Tinnitus Clinic of the Federal University of Rio de Janeiro and found no correlation between tinnitus severity and degree of hearing loss ${ }^{3}$.

In Londrina, Paraná, Brazil, 519 elderly subjects were evaluated in order to investigate the association of hearing loss and tinnitus. It was verified that,among 159 right ears studied, 58 (36.47\%) presented tinnitus, being $13(22.41 \%)$ ears with normal hearing and 45 $(77.58 \%)$ with hearing loss; and among the 159 left ears, $38(23.89 \%)$ presented tinnitus, nine (23.68\%) ears presented normal hearing and $29(76.31 \%)$ presented hearing loss ${ }^{8}$.

Although the association between tinnitus and quality of life impairment do exist, there is still little evidence to identify the specific characteristics responsible for this association, such as the factors relatedto the tinnitus complaint and the results of the audiological (hearing) evaluation - the kind and extent of hearing loss ${ }^{9}$.

Elderly people aged 60 to 80 years, from the Basic Care Coordination of Itabaiana, Sergipe, Brazil, were evaluated regarding their quality of life. Amid the 108 subjects who participated in the study, $89.1 \%$ complained of tinnitus and presented impairment in their quality of life ${ }^{10}$.
Some studies have assessed the quality of life and hearing. One of them was carried out in South Korea in order to investigate the relationship between hearing loss, tinnitus and quality of life. The sample was divided into four groups of subjects who presented normal hearing without tinnitus, normal hearing with tinnitus, hearing loss without tinnitus and hearing loss with tinnitus. The authors observed that hearing loss with tinnitus has a considerable impact on the quality of life in that population. They reported that the group of subjects with hearing loss without tinnitus had a better quality of life than the group with normal hearing and tinnitus ${ }^{11}$.

The Tinnitus Handicap Inventory - THI, an instrument that investigates the repercussion of tinnitus on subjects' quality of life, was used by researchers from the University of São Paulo. They concluded that sex and age, as well as the degree of hearing loss, did not influence the discomfort generated by tinnitus. On the other hand, the occurrence of hearing loss in individuals with tinnitus is progressively greater with the age and also in individuals with sensorineural hearing loss $^{12}$. That study aimed to evaluate the perception of the level of quality of life in subjects with tinnitus with and without hearing loss.

\section{METHODS}

This work was a cross-sectional quantitative study. It was approved by the Ethics Committee of the Universidade Tiradentes, protocol number 1.222.866.

They were included patients from the Audiology Clinic of the Department of Speech and Hearing Therapy of the institutional University Hospital with ages above 18 years, who agreed to participate in the study and signed the free and informed consent term. Exclusion criteria were: conductive or mixed hearing loss, presence of earwax or foreign body in the external ear and neurological, psychiatric or cognitive alterations already diagnosed.

In the first stage, participants answered a data collection form to identify gender, age, educational level, family income, hearing complaints and tinnitus characteristics. The second step comprisedthe evaluation of hearing, initiated by the inspection of the external acoustic meatus. The auditory evaluation was composed by conventional pure tone audiometry. Those subjects who complained of tinnitus were submitted to psychoacoustic evaluation of tinnitus through acuphenometry. The third step consisted in the application of the quality of life assessment instrument, 
the World Health Organization Quality of Life WHOQOL-BREF, and also the instrument that investigates the impact of tinnitus on the subject's quality of life, the Tinnitus Handicap Inventory - THI.

The WHOQOL-BREF instrumentmeasures thesubject's quality of life. It comprises 26 items, two of them are general questions related to the global quality of life score, and the other 24 questions are distributed in 4 domains: physical health, psychological health, social relationships, and environmental.The THI instrument investigates the repercussion of tinnitus on subjects' quality of life and is composed of 25 questions that assess the emotional, functional and catastrophic aspects.

The participants were divided into four groups, which were:

- Group 1: subjects with normal auditory thresholds without tinnitus complaint;

- Group 2: subjects both with normal auditory thresholds and tinnitus complaint;

- Group 3: subjects with sensorineural hearing loss without tinnitus complaint;

- Group 4: subjects with sensorineural hearing loss and with tinnitus complaint.

Subsequently, each group was divided again into age groups: young adults (18 to 39 years), adults (40 to 59 years) and elderly (over 60 years).
The sample consisted of 189 subjects of both genders. Age ranged from 20 to 85 years, with an average of 51.06 years $(S D= \pm 16.27)$. The $\mathrm{G} 1$ was composed of $52(27.5 \%)$ subjects, G2, composed of 43 (22.7\%) subjects, G3 by 33 (17.5\%) subjects and G4, $61(32.3 \%)$ subjects.

Statistical analyzes were performed on the software GraphPad Prism 5.0. To evaluate the influence (interaction) of the age range (age variable) in the comparisons between groups G1 to G4 (group variable), two-way analysis of variance was performed.In the case of significant interactions, data were submitted to a Shapiro-Wilk normality test, and then to the KruskallWallis test followed by Dunn's post-test for comparison between the fourgroups or Mann-Whitney test for comparison between two groups.The correlation between variables was evaluated using the Spearman correlation test, considering a level of significance of 0.05 .The descriptive analysis of the variables includedfrequency and percentage, and the age (independent variable) was analyzed by mean and standard deviation.

\section{RESULTS}

The Table 1 presents the socio-demographic data of the subjects evaluated, the auditory threshold and the presence or absence of tinnitus in at least one ear or head in the studied groups. 
Table 1. Distribution of the subjects regarding sociodemographic data, Aracaju/SE, 2015/2016

\begin{tabular}{cccccc}
\hline Studied variables & $\mathbf{G 1}$ & $\mathbf{G 2}$ & $\mathbf{G 3}$ & $\mathbf{G 4}$ & Total \\
\cline { 2 - 6 } & $\mathbf{n}(\%)$ & $\mathbf{n}(\%)$ & $\mathbf{n}(\%)$ & $\mathbf{n}(\%)$ & $\mathbf{n}(\%)$ \\
\hline Gender & & & & & \\
Female & $41(78.8)$ & $40(93.0)$ & $20(60.6)$ & $44(72.1)$ & $145(76.7)$ \\
Male & $11(21.2)$ & $3(7.0)$ & $13(39.4)$ & $17(27.9)$ & $44(23.3)$ \\
\hline Age & & & & & \\
20 to 39 years (young) & $25(48.1)$ & $17(39.5)$ & $6(18.2)$ & $4(6.6)$ & $52(27.5)$ \\
40 to 59 years (adults) & $18(34.6)$ & $19(44.2)$ & $15(45.5)$ & $25(41.0)$ & $77(40.7)$ \\
$>$ >0 years(elderly) & $9(17.3)$ & $7(16.3)$ & $12(36.4)$ & $32(52.5)$ & $60(31.8)$ \\
\hline Educational level & & & & & \\
Higher education & $13(25.0)$ & $10(23.3)$ & $3(9.1)$ & $2(3.3)$ & $28(14.8)$ \\
High school & $23(44.2)$ & $13(30.2)$ & $13(39.4)$ & $12(19.7)$ & $61(32.3)$ \\
Elementary/middle & $13(25.0)$ & $18(41.9)$ & $15(45.5)$ & $38(62.3)$ & $84(44.4)$ \\
None & $3(5.8)$ & $2(4.7)$ & $2(6.1)$ & $9(14.8)$ & $16(8.5)$ \\
\hline Family income & & & & & \\
Up to 2 minimum wages & $33(63.5)$ & $29(67.4)$ & $17(51.5)$ & $42(68.9)$ & $121(64.0)$ \\
$>$ 2 a 4 minimum wages & $8(15.4)$ & $12(27.9)$ & $14(42.4)$ & $16(26.2)$ & $50(26.5)$ \\
$>$ 4 a 10 minimum wages & $6(11.5)$ & $2(4.7)$ & $2(6.1)$ & $1(1.6)$ & $11(5.8)$ \\
$>10$ a 20 minimum wages & $3(5.8)$ & $0(0.0)$ & $0(0.0)$ & $0(0.0)$ & $3(1.6)$ \\
Not known & $2(3.8)$ & $0(0.0)$ & $0(0.0)$ & $2(3.3)$ & $4(2.1)$ \\
\hline
\end{tabular}

G1: group 1; G2: group 2; G3: group 3, G4: group 4.

The average quality of life scores were assessed according to the physical, psychological, social and environmental domains, as well as the general quality oflife.

In all domains of the WHOQOL-BREF, as well as in the aspects evaluated in the THI, no significant interactions were observed between the variables age and groups. No significant differences were observed when comparing the results among young adults, adults and the elderly.On the other hand, significant differences were observed between groups (Tables 2 and 3). These groups were compared in each domain (two-way ANOVA, $p>0.05$ ).

The comparisons between the means values of the groups in each domain of the WHOQOL-BREF are presented in Figure 1.

Table 2. Statistical data from WHOQOL-BREF per age, Aracaju/SE, 2015/2016

\begin{tabular}{cccc}
\hline Domain & $\begin{array}{c}\text { Variable: } \\
\text { Interaction age } X \text { group }\end{array}$ & Variable: age & Variable: group \\
\hline Physical health & $F 6-177=0.63 ; p=0.7054$ & $F 2-177=0.28 ; p=0.7555$ & $F 3-177=4.09 ; p=0.0077$ \\
Psychological health & $F 6-177=1.39 ; p=0.2197$ & $F 2-177=0.79 ; p=0.4563$ & $F 3-177=7.14 ; p=0.0001$ \\
Social relationships & $F 6-177=1.05 ; p=0.3930$ & $F 2-177=1.70 ; p=0.1852$ & $F 3-177=2.26 ; p=0.0828$ \\
Environmental & $F 6-177=0.93 ; p=0.4744$ & $F 2-177=2.06 ; p=0.1306$ & $F 3-177=4.24 ; p=0.0064$ \\
Perception of quality of life & $F 6-167=1.43 ; p=0.2058$ & $F 2-167=0.93 ; p=0.3969$ & $F 3-167=4.81 ; p=0.0031$ \\
Satisfaction with health & $F 6-167=1.96 ; p=0.0734$ & $F 2-167=0.37 ; p=0.6917$ & $F 3-167=5.65 ; p=0.0010$ \\
General quality of life & $F 6-167=0.92 ; p=0.4821$ & $F 2-167=1.16 ; p=0.3169$ & F3-167=6.02; $p=0.0006$ \\
\hline
\end{tabular}

Two-way analyzes of variance. 
Table 3. Statistical data from THI per age, Aracaju/SE, 2015/2016

\begin{tabular}{cccc}
\hline Domain & $\begin{array}{c}\text { Variable: } \\
\text { Interaction age } X \text { group }\end{array}$ & Variable: age & Variable: group \\
\hline Emotional & $\mathrm{F} 2-98=0.48 ; p=0.6191$ & $\mathrm{~F} 2-98=2.28 ; \mathrm{p}=0.1080$ & $\mathrm{~F} 1-98=4.29 ; \mathrm{p}=0.0410$ \\
Functional & $\mathrm{F} 2-98=0.01 ; \mathrm{p}=0.9907$ & $\mathrm{~F} 2-98=2.16 ; \mathrm{p}=0.1207$ & $\mathrm{~F} 1-98=1.25 ; \mathrm{p}=0.2653$ \\
Catastrophic & $\mathrm{F} 2-98=0.42 ; \mathrm{p}=0.6604$ & $\mathrm{~F} 2-98=1.18 ; \mathrm{p}=0.3105$ & $\mathrm{~F} 1-98=0.24 ; \mathrm{p}=0.6262$ \\
\hline
\end{tabular}

$\mathrm{THI}=$ Tinnitus Handicap Inventory; Two-way analyzes of variance.

A
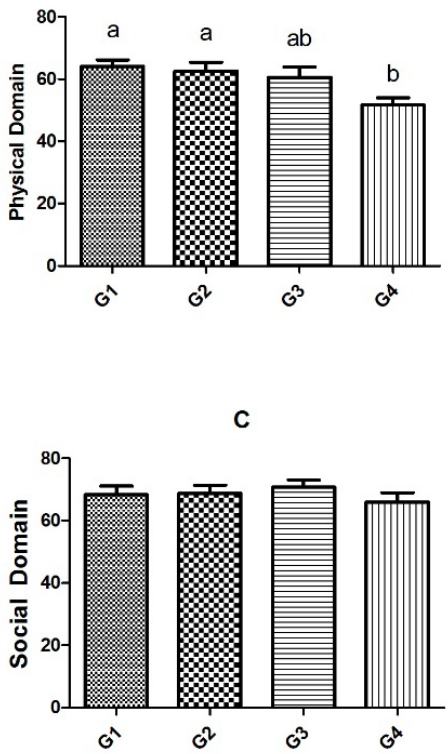

E

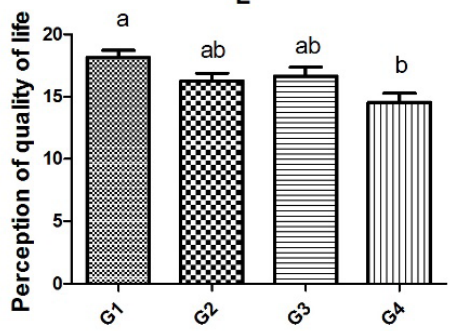

B

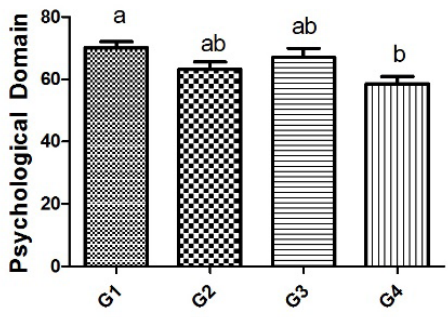

D

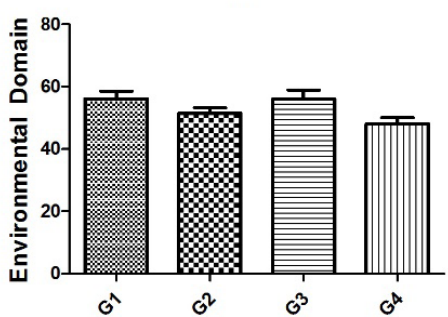

$\mathbf{F}$

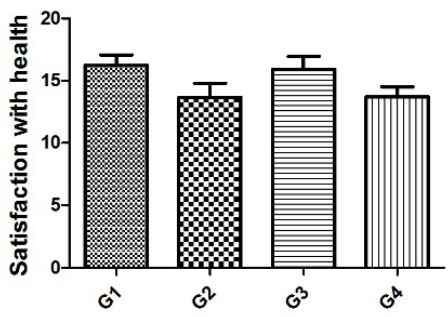

G

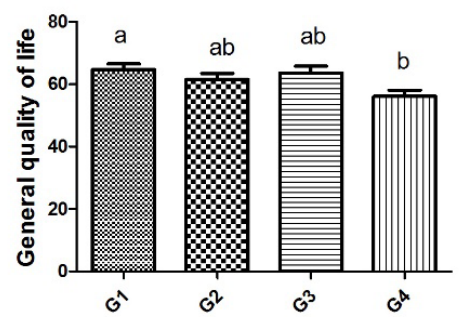

Figure 1. Distribution of the subjects regarding the scores of the WHOQOL-BREF domains: physical domain (A), psychological domain $(B)$ social domain $(C)$, environmental domain $(D)$, perception of quality of life $(E)$, satisfaction with health $(F)$, general quality of life $(G)$, subjects with normal auditory thresholds without tinnitus complaint (G1), subjects with normal auditory thresholds and tinnitus complaint (G2), subjects with sensorineural hearing loss without tinnitus complaint (G3), subjects with sensorineural hearing loss and with tinnitus complaint (G4) ). the lower letters on each bar indicate significant differences. Kruskal-Wallis test, followed by dunn's multiple comparison test. Aracaju/SE, 2015/2016 
Significant differences in the comparison between the groups were observed in the physical domain $(p=$ $0.0035), G 1$ being different from $G 4(p<0.05)$ and $G 2$ being different from $G 4(p<0.01)$ (Figure $1 A)$. Subjects with hearing loss and tinnitus have a lower quality of life score in this domain than those with normal hearing, either with or without tinnitus.

There was a significant difference in the psychological domain ( $p=0.0053), G 1$ being different from G4 ( $p$ <0.01) (Figure 1B). In this domain, subjects with normal hearingwithout complaint of tinnitus have a better average score when compared to those with hearing loss and tinnitus.

Regarding the self-assessment of quality of life perception, the groups presented significant differences ( $p=0.0026), G 1$ being different from G4 ( $p$ $<0.01$ ) (Figure 1E). This result reveals that subjects with good hearing conditions and no complaints of tinnitus evaluate their quality of life better when compared with those patients with hearing loss and tinnitus.

The overall quality of life mean scores were statistically different between the groups $(p=0.0334)$, the G4 means being lower than $\mathrm{G} 1$ ( $p<0.05$, Figure $1 G)$. Since there was no statistical difference between the groups of patientswith tinnitus (G2 and G4), it indicates that tinnitus interfere in thequality of life, associated or not with hearing loss.

They were not observed significant differences in the social $(p=0.9486$, Figure $1 C)$ and environmental ( $p=0.0771$, Figure 1D) domains, as well as in the satisfaction with health $(p=0.1172$, Figure $1 F)$.

Of the 104 subjects complaining of tinnitus, 43 (22.7\%) belonged to G2 and 61 (32.3\%) to G4. The THI scores of these groups are shown in Table 4.

Table 4. Scores from thi in groups G2 and G4, Aracaju-SE, 2015/2016

\begin{tabular}{ccccccccc}
\hline \multirow{2}{*}{ Domain in THI } & \multicolumn{9}{c}{ G2 } & \multicolumn{3}{c}{ G4 } \\
\cline { 2 - 10 } & Mean & Min & Max & SD & Mean & Min & Max & SD \\
\hline Functional & 16.79 & 0.0 & 40.0 & 12.58 & 18.65 & 0.0 & 44.0 & 12.61 \\
Emotional & 12.69 & 0.0 & 36.0 & 10.08 & 16.29 & 0.0 & 36.0 & 11.09 \\
Catastrophic & 8.65 & 0.0 & 20.0 & 38.13 & 8.91 & 0.0 & 20.0 & 6.05 \\
$\quad$ THI Total & 38.13 & 0.0 & 96.0 & 26.25 & 43.86 & 0.0 & 100.0 & 26.82 \\
\hline $\begin{array}{c}\text { Level of impairment in } \\
\text { the quality of life }\end{array}$
\end{tabular}

$\mathrm{THI}=$ Tinnitus Handicap Inventory; $\mathrm{Min}=$ minimum; $\mathrm{Max}=$ maximum; $\mathrm{SD}=$ standard deviation; $\mathrm{G}=$ group.

The analyses of the THI results by using Mann-Whitney test did not show significant differences when comparing the $\mathrm{G} 2$ and $\mathrm{G} 4$ groups in the functional $(p=0.1041)$, emotional $(p=0.4627)$, catastrophic $(p$ $=0.9152)$ and the subject's general quality of life of $(p$ $=0.2532$ ). It confirms the findings of WHOQOL-BREF, which showed that hearing loss does not interfere with the quality of life of the patients with tinnitus. In the THI, both groups presented moderate degree of discomfort to the tinnitus when the total score was evaluated, the average of G2 being 38.13 and that of G4 equal to 43.86.

The Figure 2 illustrates the correlation between the WHOQOL-BREF and THI questionnaires. Spearman's correlation test showed that there is a negative and significant correlation ( $r=-0.5570 ; p<0.0001)$, that is, the greater the discomfort caused by tinnitus, the lower the level of quality of life.

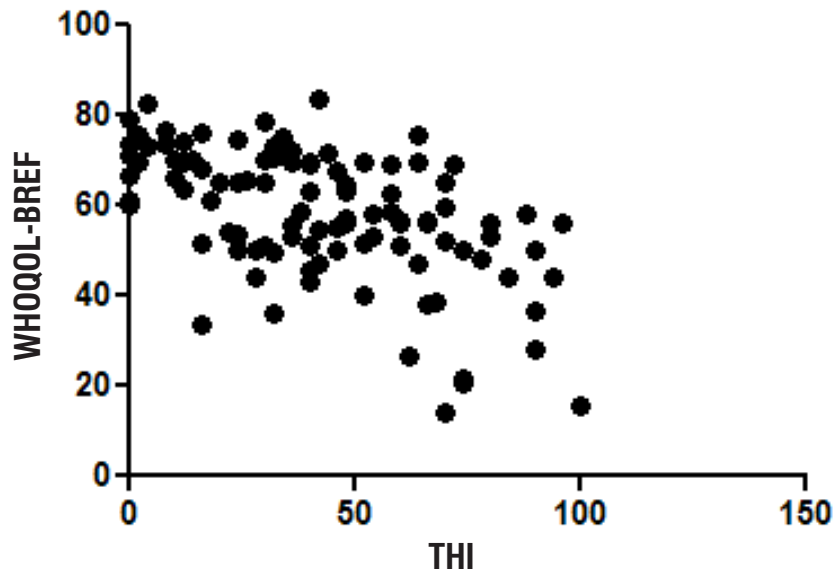

Figure 2. Correlation between WHOQOL-BREF and THI. Spearman's correlation test. Aracaju/SE, 2015-2016 
The psychoacoustic evaluation of tinnitus through acuphenometry showed the frequency range in which tinnitus was perceived by the subjects. The results in each ear are presented in Table 5, frequencies classified aslow, medium and high.

In addition, there were no significant differences when comparing the discomfortcaused by tinnitus between subjects who perceived tinnitus in the low, medium orhigh frequency ranges, for the right ear $(p$ $=0.9634)$ and for the left ear $(p=0.0834)$. Likewise, no significant differences were observed regarding thequality of life level, when compared thesubjects who perceived tinnitusin the three frequency ranges, both in the right ear $(p=0.5308)$ and the left ear $(p=0.6045)$ (Kruskal-Wallis test, followed by Dunn's multiple comparison test).

Table 5. distribution of the subjects with tinnitus (G2 and G4) by tinnitus frequency as measured in acuphenometry, Aracaju/SE, 2015/2016

\begin{tabular}{ccc}
\hline Frequency range/tone & $\begin{array}{c}\text { Right ear } \\
\mathbf{n}(\%)\end{array}$ & $\begin{array}{c}\text { Left ear } \\
\mathbf{n}(\%)\end{array}$ \\
\hline Low $(125$ to $750 \mathrm{~Hz})$ & $16(25.0)$ & $16(22.8)$ \\
Medium $(1000$ to $300 \mathrm{~Hz})$ & $13(20.3)$ & $11(15.8)$ \\
High $(4000$ to $8000 \mathrm{~Hz})$ & $35(54.7)$ & $43(61.4)$ \\
\hline Total & $64(100.0)$ & $70(100.0)$ \\
\hline
\end{tabular}

\section{DISCUSSION}

The analysis of the population studied revealed a largest participation of women, which is in agreement with previous studies ${ }^{3,13}$. This data probably results from the fact that women are more careful about their own health, seeking medical attention more often when compared to men.

The predominant educational level in the studied population was the primary education, which is probably related to low family income. Generally the population with a low socioeconomic level is the one that most seeks public health services, and the studied population herewas coming from a public University Hospital.It was verified that most of the subjects reported receiving up to two minimum wages, what is in agreement with another study performed with subjects attended in the primary care of the cities of Rio de Janeiro and São Paulo ${ }^{13}$.

Since there were no statistically differences when compared the results of THI and WHOQOL-BREF considering different ages (young, adults and elderly), but the differences remain among groups, it was considered that tinnitus interferes in the general quality of life, independently of the age or the presence of associated hearing loss. This result demonstrates how much the tinnitus bothers people's daily life, even more than the discomfortgenerated by the difficulty to listen to the sounds of the environment or to understand the conversations. The mean scores of quality of life were statically lower in the G4 than in G1, what is agree with other authors who reported an important impact of tinnitus with hearing loss in a Korean population ${ }^{11}$. They also verified that the group with tinnitus without hearing loss had a better level of quality of life than the group with normal hearing and tinnitus, what indicates that tinnitus has a closer relationship with quality of life than hearing loss ${ }^{11}$.

When assessing the psychological domain, it was verified that subjects with normal hearing without complaint of tinnitus have a higher average score when compared to those who have hearing loss and tinnitus, showing that alterations in the auditory system affect the psychic of individuals.

There was no statistical difference between groups in the environmental domain, and all of them presented low scores. This result indicates that the issues assessed in this area, such as physical security, home environment, financial resources, health and social care, opportunities to acquire information and skills, opportunities of leisure and participation in recreational activities, physical environment (pollution/noise/ traffic/climate) and transport, are unsatisfactory for all studied population.It should be emphasized that the population studied comes from a public health service, which usually has a low socioeconomic level, and it may be associated with the issues evaluated in the environmental domain -the domain with a lower score 
- thus indicating a lack of housing conditions, basic sanitation and transportation, access to the media, health services, leisure and general information. Other authors found similar results regarding the low score in the environmental domain ${ }^{13,14}$.

When analyzing the THI instrument, it was observed that G2 and G4 presented a moderate-level ofdiscomfort with tinnitus, which means that tinnitus is perceived in the presence of a background noise, although daily activities can still be performed. Some authors also found mild discomfort to tinnitus in similar studies ${ }^{15,16}$ while others different results ${ }^{3,17}$. It reveals that tinnitus interferes in subjects' quality of life independently of having preserved or altered auditory thresholds.

The significant correlation found between the data analyzed revealed that the higher the subject's perception of tinnitus, the lower the generalquality of life level. Previous works have shown that the high frequency tinnitus was reported by most of subjects ${ }^{18-20}$. Another study found the highest occurrence of tinnitus in the medium frequencies ${ }^{15}$. The present study corroborates previous works regarding the occurrence of high frequency of tinnitus, but it was not observed relationships between the frequency range and the discomfort generated by to tinnitus. Therefore, it was verified that patients with tinnitus were uncomfortable with this symptom regardless they perceive it as low, medium or high.Likewise, the quality of life level was not influenced by the frequency range of tinnitus. Similar result was found in a previous work ${ }^{15}$, however a correlation between frequency range and discomfort to tinnitus was observed: the higher the tinnitus frequency, the higher the THI score ${ }^{18}$.

It is important to alert the population about the interference of tinnitus in the level of quality of life, as well as to develop means to reduce the discomfort caused by this symptom, then improving the quality of life of the subjects.

\section{CONCLUSION}

Tinnitus interferes with the quality of life of individuals who have preserved or altered auditory thresholds. The subjects complained of tinnitus presented a moderate degree of discomfort to this symptom. Age did not influence subjects' quality of life. Those patients with sensorineural hearing loss and tinnitus complaint presented lower mean scores of quality of life than those with normal auditory thresholds without tinnitus complaint.

\section{REFERENCES}

1. Figueiredo RR, Azevedo AA. Introdução, epidemiologia e classificações. In: Figueiredo RR, Azevedo AA (orgs). Zumbido. Rio de Janeiro: Revinter, 2013. p. 1-4.

2. Santoshi Kumari M, Madhavi J, Meganadh KR, Jyothy A. Association of tinnitus and hearing loss in ontological disorders: a decade-long epidemiological study in a South Indian population. Braz. j. otorhinolaryngol. 2016;82(6):643-9.

3. Pinto PC, Sanchez TG, Tomita S. The impact of gender, age and hearing loss on tinnitus severity. Braz. j. otorhinolaryngol. 2010;76(1):1824.

4. Kochkin S, Tyler R, Born J. Marketrak VIII: The prevalence of tinnitus in the United States and the self-reported efficacy of various treatments. Hearing Review. 2011;18(12):10-27.

5. Bhatt JM, Lin HW, Bhattacharyya N. Prevalence, severity, exposures and treatment patterns of tinnitus in the United States. JAMA Otolaryngol Head Neck Surg. 2016;142(10):959-65.

6. Oiticica J, Bittar RSM. Tinnitus prevalence in the city of São Paulo. Braz. j. otorhinolaryngol. 2015;81(2):167-76.

7. Martinez C, Wallenhorst C, McFerran D, HallL DA. Incidence rates of clinically significant tinnitus: 10-year trend from a cohort study in England. Ear Hear. 2015;36(3):69-75.

8. Gibrin PCD, Melo JJ, Marchioril LLM. Prevalence of tinnitus complaints and probable association with hearing loss, diabetes mellitus and hypertension in elderly. CoDAS. 2013;25(2):176-80.

9. Weidt S, Delsignore A, Meyer M, Rufer M, Peter N, Drabe $\mathrm{N}$ et al. Which tinnitus-related characteristics affect current health-related quality of life and depression? A cross-sectional cohort study. Psychiatry Rsearch. 2016;237:114-21.

10. Gois RO, Gois BO, Pereira MCCS, Taguchi CK. State mental and impact of the tinnitus in the elderly. Rev. CEFAC. 2014;16(3):798-809.

11. Joo Y, Han K, Park KH. Association of hearing loss and tinnitus with health-related quality of life: The Korea National Health and Nutrition Examination Survey. PLOS ONE. 2015;10(6):1-10.

12. Mondelli MFCG, Rocha AB. Correlation between the audiologic findings and buzz disturbing. Arq. Int. Otorrinolaringoly. 2011;15(2):172-80.

13. Portugal FB, Campos MR, Gonçalves DA, Mari JJ, Fortes SLCL. Quality of life of primary care patients in Rio de Janeiro and São Paulo, Brasil: associations 
with stressful life events and mental health. Ciência \& Saúde Coletiva. 2016;21(2):497-508.

14. Olivares A, Bonito J, Silva R. Qualidade de vida no trabalho dos médicos da atenção básica no estado de Roraima (BRASIL). Psicologia, Saúde e Doenças. 2015;16(1):100-11.

15. Cribari J, Ribas A, Fonseca VR, Moretti CM, Zeigelboim BS, Martins $\mathrm{J}$ et al. Description of tinnitus and its relation to discomfort level in a group of elderly hearing aid wearers. Int Tinnitus J. 2016;20(1):36-41.

16. Lacerda ABN, Facco C, Zeigelboim BS, Cristoff $\mathrm{K}$, Stechman Neto J, Fonseca VR. The impact of tinnitus on the quality of life in patients with temporomandibular dysfunction. Int Tinnitus J. 2016;20(1):24-30.

17. Cardoso NA, Hoshino ACH, Perez MA, Bastos WR, Carvalho DP, Câmara VM. Tinnitus in a riverside population exposed to methyl-mercury. Audiol Commun Res. 2014;19(1):40-4.

18. Morais AA, Gil D. Tinnitus in individuals without hearing loss and its relationship with temporomandibular dysfunction. Braz. j. otorhinolaryngol. 2012;78(2):59-65.

19. Seimetz BM, Teixeira AR, Rosito LPS, Flores LS, Pappen $\mathrm{CH}$, Dall'igna C. Pitch and loudness tinnitus in individuals with presbycusis. Int Arch Otorhinolaryngol. 2016;20(4):321-6.

20. Gentil F, Meireles S, Roza T, Santos C, Parente $M$, Almeida $E$ et al. Comparison of otoacoustic emissions in patients with tinnitus having normal hearing versus mild hearing loss. Int Tinnitus $\mathrm{J}$. 2015;19(2):39-46. 